\title{
Front Matter: Volume 7599
}

, "Front Matter: Volume 7599," Proc. SPIE 7599, Organic Photonic Materials and Devices XII, 759901 (1 April 2010); doi: 10.1117/12.862671

SPIE. Event: SPIE OPTO, 2010, San Francisco, California, United States 


\section{PROCEEDINGS OF SPIE}

\section{Organic Photonic Materials and Devices XII}

Robert L. Nelson

François Kajzar

Toshikuni Kaino

Editors

26-28 January 2010

San Francisco, California, United States

Sponsored and Published by

SPIE

Volume 7599 
The papers included in this volume were part of the technical conference cited on the cover and title page. Papers were selected and subject to review by the editors and conference program committee. Some conference presentations may not be available for publication. The papers published in these proceedings reflect the work and thoughts of the authors and are published herein as submitted. The publisher is not responsible for the validity of the information or for any outcomes resulting from reliance thereon.

Please use the following format to cite material from this book:

Author(s), "Title of Paper," in Organic Photonic Materials and Devices XII, edited by Robert L. Nelson, François Kajzar, Toshikuni Kaino, Proceedings of SPIE Vol. 7599 (SPIE, Bellingham, WA, 2010) Article CID Number.

ISSN 0277-786X

ISBN 9780819479952

Published by

SPIE

P.O. Box 10, Bellingham, Washington $98227-0010$ USA

Telephone +1 3606763290 (Pacific Time) · Fax +1 3606471445

SPIE.org

Copyright (@ 2010, Society of Photo-Optical Instrumentation Engineers

Copying of material in this book for internal or personal use, or for the internal or personal use of specific clients, beyond the fair use provisions granted by the U.S. Copyright Law is authorized by SPIE subject to payment of copying fees. The Transactional Reporting Service base fee for this volume is $\$ 18.00$ per article (or portion thereof), which should be paid directly to the Copyright Clearance Center (CCC), 222 Rosewood Drive, Danvers, MA 01923. Payment may also be made electronically through CCC Online at copyright.com. Other copying for republication, resale, advertising or promotion, or any form of systematic or multiple reproduction of any material in this book is prohibited except with permission in writing from the publisher. The CCC fee code is 0277-786X/10/\$18.00.

Printed in the United States of America.

Publication of record for individual papers is online in the SPIE Digital Library.

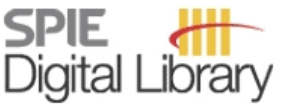

SPIEDigitalLibrary.org

Paper Numbering: Proceedings of SPIE follow an e-First publication model, with papers published first online and then in print and on CD-ROM. Papers are published as they are submitted and meet publication criteria. A unique, consistent, permanent citation identifier (CID) number is assigned to each article at the time of the first publication. Utilization of CIDs allows articles to be fully citable as soon they are published online, and connects the same identifier to all online, print, and electronic versions of the publication. SPIE uses a six-digit CID article numbering system in which:

- The first four digits correspond to the SPIE volume number.

- The last two digits indicate publication order within the volume using a Base 36 numbering system employing both numerals and letters. These two-number sets start with 00, 01, 02, 03, 04 , 05, 06, 07, 08, 09, OA, OB ... 0Z, followed by 10-1Z, 20-2Z, etc.

The CID number appears on each page of the manuscript. The complete citation is used on the first page, and an abbreviated version on subsequent pages. Numbers in the index correspond to the last two digits of the six-digit CID number. 


\section{Contents}

vii Conference Committee

PHOTONIC TECHNOLOGIES

759905 Complementary grating dynamics in photorefractive polymers with Alq 3 [7599-04]

C. W. Christenson, J. Thomas, P.-A. Blanche, R. Voorakaranam, R. A. Norwood, College of Optical Sciences, The Univ. of Arizona (United States); M. Yamamoto, Nitto Denko Technical Corp. (United States); N. Peyghambarian, College of Optical Sciences, The Univ. of Arizona (United States)

\section{ORGANIC NANOPHOTONICS}

759909 Controlling emission color through Förster resonance energy transfer in hybrid colloidal particles [7599-08]

C. F. Huebner, S. H. Foulger, Clemson Univ. (United States)

7599 OA Piecewise fabrication of click functionalized core-shell particles [7599-09]

R. D. Roeder, D. D. Evanoff, Jr., S. H. Foulger, Clemson Univ. (United States)

\section{ELECTRO-OPTICS I}

7599 OG Hyper-branched polymer for electro-optic applications (Invited Paper) [7599-15]

X. Piao, Y. Mori, X. Zhang, S. Inove, S. Yokoyama, Kyushu Univ. (Japan)

$7599 \mathrm{OH} \quad$ EO polymer modulators reliability study [7599-16]

D. Jin, H. Chen, A. Barklund, J. Mallari, G. Yu, E. Miller, R. Dinu, GigOptix, Inc. (United States)

7599 ol High-performance electro-optic modulators realized with a commercial side-chain DR1-PMMA electro-optic copolymer (Invited Paper) [7599-17]

S. Michel, J. Zyss, I. Ledoux-Rak, C. T. Nguyen, Lab. de Photonique Quantique et Moléculaire, CNRS, École Normale Supérieure de Cachan (France)

\section{ELECTRO-OPTICS II}

7599 OK Hybrid organic crystal/silicon-on-insulator integrated electro-optic modulators (Invited Paper) [7599-19]

M. Jazbinsek, C. Hunziker, S.-J. Kwon, H. Figi, O.-P. Kwon, P. Günter, ETH Zürich (Switzerland)

7599 OL Organically enabled silicon-based photonic/RF-photonic applications [7599-20]

A. Sharkawy, O. Ebil, P. Yao, E. Kelmelis, EM Photonics, Inc. (United States); M. Zablocki, S. Shi,

C. A. Schuctz, D. W. Prather, Univ. of Delaware (United States) 


\section{ACTIVE MATERIALS}

7599 OR Optical, photoluminescent, and photoconductive properties of functionalized anthradithiophene and benzothiophene derivatives [7599-25]

W. E. B. Shepherd, A. D. Platt, G. Banton, Oregon State Univ. (United States); M. A. Loth, J. E. Anthony, Univ. of Kentucky (United States); O. Ostroverkhova, Oregon State Univ. (United States)

\section{WAVEGUIDE DEVICES}

7599 OT Development of print-like-fabrication techniques for distributed feedback solid state dye lasers with multiple-layered structure [7599-27]

S. Omi, N. Kamogawa, H. Watenabe, Y. Yang, Y. Oki, Kyushu Univ. (Japan)

7599 OU The effect of trans-stilbene unit in the compensation of birefringence of poly(methyl methacrylate) in the random copolymerization method and anisotropic molecule dopant method [7599-28]

H. Shafiee, A. Tagaya, Y. Koike, Keio Univ. (Japan) and Japan Science and Technology Agency (Japan)

\section{NONLINEAR ABSORPTION I}

7599 OW Maximizing two-photon absorption cross section within few essential state model [7599-30] A. Rebane, M. Drobizhev, N. S. Makarov, E. Beuerman, Montana State Univ. (United States); Y. Zhao, C. W. Spangler, SensoPath Technologies, Inc. (United States)

7599 0X Justification of two-level approximation for description of two-photon absorption in oxazine dyes [7599-31]

E. Beverman, N. S. Makarov, M. Drobizhev, Montana State Univ. (United States); A. Rebane, Montana State Univ. (United States) and National Institute of Chemical Physics and Biophysics (Estonia)

\section{NEW MATERIALS AND METHODS}

759912 Novel highly emissive tetracyanotetraphenylporphyrazine ytterbium complex for optoelectronic and biophotonic applications [7599-36]

L. G. Klapshina, G.A. Razuvaev Institute of Organometallic Chemistry (Russian Federation);

W. E. Douglas, Lab. CMOS, CNRS, Institut Gerhardt, Univ. Montpellier II (France); I. S. Grigoryev, G.A. Razuvaev Institute of Organometallic Chemistry (Russian Federation); M. V. Shirmanova, Nizhny Novgorod State Medical Academy (Russian Federation);

P. Gerbier, Lab. CMOS, CNRS, Institut Gerhardt, Univ. Montpellier II (France); B. A. Bushuk, B.I. Stepanov Institute of Physics (Belarus) 
759914 Photostability enhancement studies on zwitterionic chromophores for nonlinear optics [7599-38]

S. G. Raymond, S. Janssens, My. T. T. Do, G. V. M. Williams, N. Denton, J. Anderson, M. D. H. Bhuiyan, Industrial Research Ltd. (New Zealand)

$759915 \quad$ Nonlinear transmission using highly nonlinear Bragg mirrors [7599-39]

X. Zhu, The Univ. of Arizona (United States); J. Wang, The Univ. of Arizona (United States) and TIPD LLC (United States); P. Lau, D. Nguyen, R. A. Norwood, The Univ. of Arizona (United States); D. Steeves, B. Kimball, U.S. Army Natick Soldier Ctr. (United States); N. Peyghambarian, The Univ. of Arizona (United States) and TIPD LLC (United States)

\section{NONLINEAR ABSORPTION II}

759918 Novel nonlinear transmission of porphyrin complexes containing rhenium selenide clusters [7599-42]

J. Wang, College of Optical Sciences, The Univ. of Arizona (United States) and TIPD LLC (United States); X. Zhu, College of Optical Sciences, The Univ. of Arizona (United States); X. Tu, Z. Zheng, The Univ. of Arizona (United States); R. Norwood, College of Optical Sciences, The Univ. of Arizona (United States); D. Steeves, B. Kimball, U.S. Army Natick Soldier Ctr. (United States); N. Peyghambarian, College of Optical Sciences, The Univ. of Arizona (United States) and TIPD LLC (United States)

759919 Modeling of non-Lorentzian two-photon absorption line shape in dipolar chromophore [7599-43]

A. K. Rebane, Montana State Univ. (United States) and National Institute of Chemical Physics and Biophysics (Estonia); M. Drobizhev, N. S. Makarov, E. Beverman, Montana State Univ. (United States); C. Nacke, J. Pahapill, National Institute of Chemical Physics and Biophysics (Estonia)

\section{APPLICATIONS}

7599 ID Wavelength resolution improvement on organic photodiodes made by ink-jet technique [7599-47]

Y. Yang, T. Nakamichi, S. Omi, R. Goto, Kyushu Univ. (Japan); M. Yahiro, Institute of Systems, Information Technologies, and Nanotechnologies (Japan); M. Era, Saga Univ. (Japan);

H. Watanabe, Y. Oki, Kyushu Univ. (Japan)

7599 IE Optofluidic distributed feedback dye laser via evanescent gain [7599-48]

W. Song, A. E. Vasdekis, D. Psaltis, Ecole Polytechnique Fédérale de Lausanne (Switzerland)

\section{POSTER SESSION}

7599 IG Molecular orientation of discotic molecules controlled using self-assembled monolayer films [7599-50]

C.-Y. Chiang, Y.-T. Hu, C. W. Ong, W. Zheng, National Sun Yat-Sen Univ. (Taiwan)

7599 IK Color tuning of photonic gel films by UV irradiation [7599-55] 
S. E. Shin, S. Y. Kim, D. M. Shin, Hongik Univ. (Korea, Republic of)

7599 IL Anchoring energy of photo-sensitive polyimide alignment film containing methoxy cinnamate [7599-56]

S. Kim, S. E. Shin, D. M. Shin, Hongik Univ. (Korea, Republic of)

7599 1M Improvement in lifetime of green organic light-emitting device [7599-57]

H. C. Ki, S. H. Kim, D. G. Kim, H. J. Kim, H. Ko, M.-S. Han, H. J. Kim, Korea Photonics Technology Institute (Korea, Republic of); K. J. Hong, Gwangju Univ. (Korea, Republic of)

7599 iN Integrated electro-optic devices of melt-processable single-crystalline organic films [7599-58]

H. Figi, M. Jazbinšek, C. Hunziker, M. Koechlin, P. Günter, ETH Zürich (Switzerland)

759910 Picosecond and nanosecond third order nonlinear optical characterization of $\mathrm{Cu}$ and $\mathrm{Ni}$ phthalocyanines using Z-scan technique [7599-59]

S. V. Rao, Univ. of Hyderabad (India); A. K. Prasad, Cochin Univ. of Science and Technology (India); L. Giribabu, Indian Institute of Commerce and Trade (India); S. P. Tewari, Univ. of Hyderabad (India)

7599 IP Pump-probe experiments with sub-100 femtosecond pulses for characterizing the excited state dynamics of phthalocyanine thin films [7599-60]

S. V. Rao, D. Swain, S. P. Tewari, Univ. of Hyderabad (India)

7599 IR Comparative study on the effect of thermal annealing on polymer/small molecule blend and copolymer light-emitting devices [7599-62]

P. Rungta, V. Tsyalkovsky, Y. P. Bandera, M. A.-A. Daniele, S. H. Foulger, Clemson Univ. (United States)

Author Index 


\section{Conference Committee}

Symposium Chair

E. Fred Schubert, Rensselaer Polytechnic Institute (United States)

Symposium Cochairs

Liang-Chy Chien, Kent State University (United States)

James G. Grote, Air Force Research Laboratory (United States)

Program Track Chair

James G. Grote, Air Force Research Laboratory (United States)

Conference Chairs

Robert L. Nelson, Air Force Research Laboratory (United States)

François Kajzar, Université d'Angers (France)

Toshikuni Kaino, Tohoku University (Japan)

Program Committee

Chantal Andraud, École Normale Supérieure de Lyon (France)

Werner J. Blau, Trinity College Dublin (Ireland)

Christoph Bubeck, Max-Planck-Institut für Polymerforschung (Germany)

Darnell E. Diggs, Air Force Research Laboratory (United States)

Alain F. Fort, Institut de Physique et Chimie des Matériaux de Strasbourg (France)

James G. Grote, Air Force Research Laboratory (United States)

F. Kenneth Hopkins, Air Force Research Laboratory (United States)

Alex K.-Y. Jen, University of Washington (United States)

Michael H. C. Jin, The University of Texas at Arlington (United States)

Eunkyoung Kim, Yonsei University (Korea, Republic of)

Jang-Joo Kim, Seoul National University (Korea, Republic of)

Nakjoong Kim, Hanyang University (Korea, Republic of)

Junya Kobayashi, NTT Photonics Laboratory (Japan)

Isabelle N. Ledoux-Rak, École Normale Supérieure de Cachan

(France)

Charles Y. C. Lee, Air Force Office of Scientific Research (United States)

Kwang-Sup Lee, Hannam University (Korea, Republic of)

Emisoon Mah, Air Force Research Laboratory (United States)

Seth R. Marder, Georgia Institute of Technology (United States)

Antoni Cz. Mitus, Wrocław University of Technology (Poland)

Robert A. Norwood, College of Optical Sciences, The University of

Arizona (United States)

Jean-Michel Nunzi, Queen's University (Canada) 
Susanna Orlic, Technische Universität Berlin (Germany)

Ileana Rau, Politehnica University of Bucharest (Romania)

Niyazi Serdar Sariciftci, Johannes Kepler Universität Linz (Austria)

Kenneth D. Singer, Case Western Reserve University (United States)

Don J. Smith, U.S. Air Force (United Kingdom)

Rebecca E. Taylor, Lockheed Martin Space Systems Co. (United States)

Toshiyuki Watanabe, Tokyo University of Agriculture and Technology (Japan)

\section{Session Chairs}

1 Photonic Technologies

Robert L. Nelson, Air Force Research Laboratory (United States)

2 Organic Nanophotonics

Toshikuni Kaino, Tohoku University (Japan)

3 NLO Materials and Devices I

Yasuhiro Koike, Keio University (Japan)

4 Electro-Optics I

Toshikuni Kaino, Tohoku University (Japan)

5 Electro-Optics II

Robert L. Nelson, Air Force Research Laboratory (United States)

6 Active Materials

Robert A. Norwood, College of Optical Sciences, The University of Arizona (United States)

$7 \quad$ Waveguide Devices

Shiyoshi Yokoyama, Kyushu University (Japan)

8 Nonlinear Absorption I

Ileana Rau, Politehnica University of Bucharest (Romania)

9 New Materials and Methods

Chantal Andraud, Ecole Normale Supérieure de Lyon (France)

10 NLO Materials and Devices II

Fabrice Charra, Commissariat à l'Énergie Atomique (France)

11 Nonlinear Absorption II

Kwang-Sup Lee, Hannam University (Korea, Republic of)

12 Applications

François Kajzar, Université d'Angers (France) 